\title{
Diode-Triggered Silicon-Controlled Rectifier With Reduced Voltage Overshoot for CDM ESD Protection
}

\author{
Wen-Yi Chen, Student Member, IEEE, Elyse Rosenbaum, Fellow, IEEE, and Ming-Dou Ker, Fellow, IEEE
}

\begin{abstract}
Diode-triggered silicon-controlled rectifiers (DTSCRs) are used for on-chip electrostatic discharge protection. The role of the trigger diode string in determining the transient voltage overshoot is investigated using a very fast transmission line pulse. A DTSCR containing only poly-bound trigger diodes has a voltage overshoot of just $1.5 \mathrm{~V}$ at $7 \mathrm{~A}$, which is significantly less than what is found with STI-bound diodes. A DTSCR with only STI-bound trigger diodes has a lower leakage current. Therefore, DTSCRs with different trigger diode configurations may be suitable for different applications, e.g., high speed or low power.
\end{abstract}

Index Terms-Charge device model (CDM), electrostatic discharge (ESD), silicon-controlled rectifier (SCR).

\section{INTRODUCTION}

$\mathbf{S}$ ILICON-CONTROLLED RECTIFIER (SCR) devices safely handle high current densities, making them attractive for electrostatic discharge (ESD) protection [1]. Various designs have been proposed to further improve the ESD protection efficiency of SCR. For example, the structure in [2] utilizes a dummy-gate structure to improve the turn-on speed of the SCR device. Among the various SCR-based ESD protection designs, the diode-triggered SCR (DTSCR) prevails in advanced CMOS technologies due to its design simplicity [3], [4]. When SCR-based protection devices are subject to nanosecond-scale discharges, such as charged device model (CDM) ESD, they are often unable to clamp the pad voltage below the breakdown voltage of thin gate oxides, particularly in sub-100-nm CMOS technologies [5]-[10]. Although the holding voltage of a typical SCR is about $1.5 \mathrm{~V}$, the device cannot be switched instantly from off to on; if the applied ESD pulse has a subnanosecond

Manuscript received July 19, 2011; revised September 7, 2011 and September 26, 2011; accepted October 1, 2011. Date of publication October 13, 2011; date of current version March 7, 2012. This work was supported in part by the National Science Council, Taiwan, under Contract NSC 98-2221-E-009-113-MY2 and in part by the "Aim for the Top University Plan" of National Chiao Tung University and the Ministry of Education, Taiwan. The work of W.-Y. Chen was supported by the Graduate Students Study Abroad Fellowship from the National Science Council, Taiwan. The work of E. Rosenbaum was supported by the SRC.

W.-Y. Chen is with the Institute of Electronics, National Chiao Tung University, Hsinchu 300, Taiwan.

E. Rosenbaum is with the Department of Electrical and Computer Engineering, University of Illinois at Urbana-Champaign, Urbana, IL 61801 USA.

M.-D. Ker is with the Department of Electronics Engineering, National Chiao Tung University, Hsinchu 300, Taiwan, and also with the Department of Electronic Engineering, I-Shou University, Kaohsiung 840, Taiwan (e-mail: mdker@ieee.org).

Color versions of one or more of the figures in this paper are available online at http://ieeexplore.iee.org.

Digital Object Identifier 10.1109/TDMR.2011.2171487
TABLE I

TEST STRUCTURES STUdied IN THIS WORK

\begin{tabular}{|c|c|c|c|}
\hline & $\begin{array}{c}\text { Acronym in } \\
\text { Text / Figures }\end{array}$ & $\begin{array}{c}\text { Type of Diode } \\
\text { Intrinsic to the SCR }\left(\mathrm{D}_{1}\right)\end{array}$ & $\begin{array}{c}\text { Type of External Trigger } \\
\text { Diodes }\left(\mathrm{D}_{2}, \mathrm{D}_{3} \text {, and } \mathrm{D}_{4}\right)\end{array}$ \\
\hline A & STI-DTSCR & STI-bound & all STI-bound \\
\hline B & Diode-String & $\begin{array}{c}\text { STI-bound (no SCR action, } \\
\text { SCR cathode removed) }\end{array}$ & all STI-bound \\
\hline C & Mix-DTSCR & STI-bound & all Poly-bound \\
\hline D & Poly-DTSCR & Poly-bound & all Poly-bound \\
\hline
\end{tabular}

rise time, a transient voltage overshoot is observed [11], and it requires that the SCR be augmented by an additional protection element [12], [13]. Before the SCR fully turns on, the voltage across its terminals is determined by the trigger circuit [14]. This observation suggests that one should employ a trigger circuit that turns on quickly to provide good voltage clamping.

At high frequencies, diodes exhibit inductive characteristics as a result of conductivity modulation [15]. This indicates that the diode string trigger circuit of a DTSCR will itself display some transient voltage overshoot [14]. In a given technology node, poly-bound diodes [16] have smaller voltage overshoot than do STI-bound diodes, a result of the shorter base region [17]. It follows that a DTSCR built using poly-bound diodes should provide better voltage clamping than would one built using STI-bound diodes. This work evaluates that conjecture by means of experiments performed on DTSCR devices fabricated in a 65-nm low-power CMOS technology.

\section{EXPERIMENT}

The test structures used in this work are listed in Table I. The DTSCRs all contain three external $\mathrm{p}^{+} / \mathrm{n}$-well diodes, labeled as $\mathrm{D}_{2}, \mathrm{D}_{3}$, and $\mathrm{D}_{4}$ in Fig. $1(\mathrm{a}) . \mathrm{D}_{2}, \mathrm{D}_{3}$, and $\mathrm{D}_{4}$ each have a p-n junction perimeter of $200 \mu \mathrm{m}$. In test structure A (STI-DTSCR), these are STI-bound diodes with a $\mathrm{p}^{+}$-to-n ${ }^{+}$spacing of $360 \mathrm{~nm}$. Test structure B (Diode-String) is similar to A, except that the $\mathrm{n}^{+}$cathode of the SCR has been removed, disabling the SCR and allowing one to observe the characteristics of just the diode string. In test structure $\mathrm{C}$ (Mix-DTSCR), $\mathrm{D}_{2}, \mathrm{D}_{3}$, and $\mathrm{D}_{4}$ are poly-bound diodes with a $\mathrm{p}^{+}$-to-n ${ }^{+}$spacing (gate width) of $65 \mathrm{~nm}$. Test structure D (Poly-DTSCR) is similar to C, except that $\mathrm{D}_{1}$, the trigger diode integrated within the SCR, has been changed from STI-bound to poly-bound. The cross section of a Poly-DTSCR is shown in Fig. 1(b).

In all cases, the SCR has an effective width of $50 \mu \mathrm{m}$; this also means that $\mathrm{D}_{1}$ has a $50-\mu \mathrm{m}$ perimeter. The SCR 


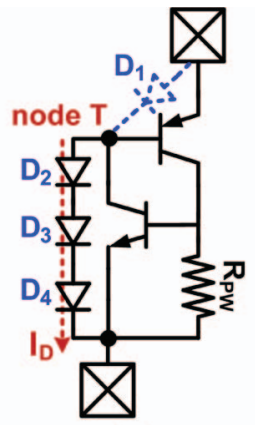

(a)

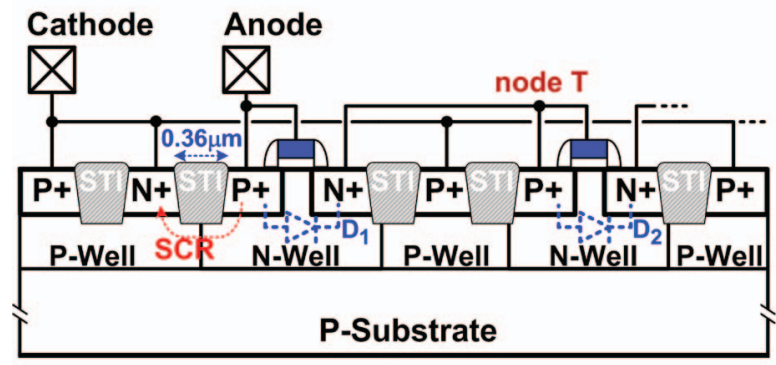

(b)

Fig. 1. (a) Schematic representation of the DTSCRs used in this work and (b) cross section of a Poly-DTSCR.

anode-to-cathode spacing $\left(S_{\mathrm{ac}}\right)$ was fixed at the minimum value allowed by the layout design rules, which is $0.36 \mu \mathrm{m}$, so as to minimize the intrinsic turn-on delay of the SCR [4].

A very-fast transmission line pulse (vf-TLP) system is used to generate pulses with a pulse width of $5 \mathrm{~ns}$ and a rise time of 100 ps [18], [19]. These pulses are applied to the DTSCRs, and the device response is recorded using a $12-\mathrm{GHz}$ oscilloscope.

\section{VF-TLP RESULTS AND DISCUSSION}

A single quasi-static $I-V$ point is obtained from the vf-TLP data by averaging the current and voltage over a 500-ps interval, starting 3 ns after the pulse rising edge (see Fig. 3). The resulting $I-V$ curves are shown in Fig. 2(a). There are three regions in the quasi-static $I-V$ curve: (I) SCR is off, and $\overline{V_{\text {DUT }}}<V_{t 1}$, where $V_{t 1}$ is the on-voltage of the diode string; (II) SCR is on; and (III) SCR is on, and the diode string is also on, because $\overline{V_{\text {DUT }}}>V_{t 1}$. In regions $\mathrm{I}$ and II, the three DTSCRs have nearly identical quasi-static $I-V$ characteristics. In region III, where the SCR and the diode string provide parallel paths for the ESD current, the Poly-DTSCR has lower on-resistance than the other two DTSCRs because the poly-bound diodes have a smaller static $R_{\text {on }}$ than the STI-bound diodes.

In Fig. 2(b), the current is instead plotted as a function of the peak voltage appearing across the device under test (DUT). The STI-DTSCR and the Diode-String have nearly identical $I-V$ characteristics, at least for $V_{\text {peak }}<13 \mathrm{~V}$, highlighting that $V_{\text {peak }}$ is determined by the trigger circuit, not the SCR. Changing the SCR-based structure from STI-DTSCR to PolyDTSCR dramatically reduces the amount of voltage overshoot $\left(V_{\mathrm{OV}}\right)$, not only because the poly-bound diodes have a lower static $R_{\text {on }}$ than STI-bound diodes but also because they display less inductive behavior.

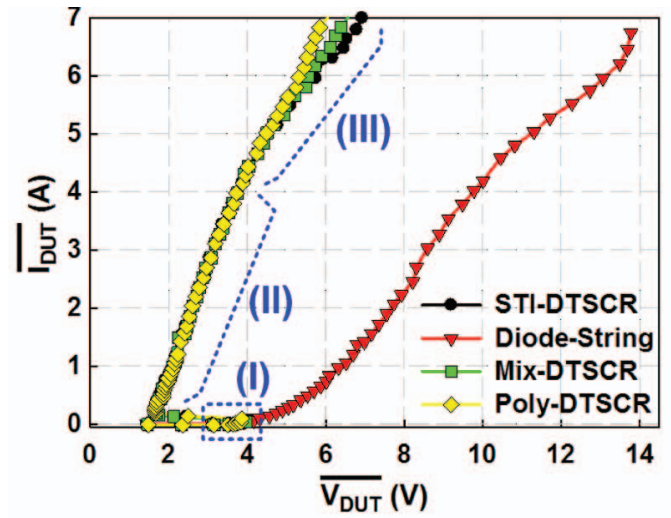

(a)

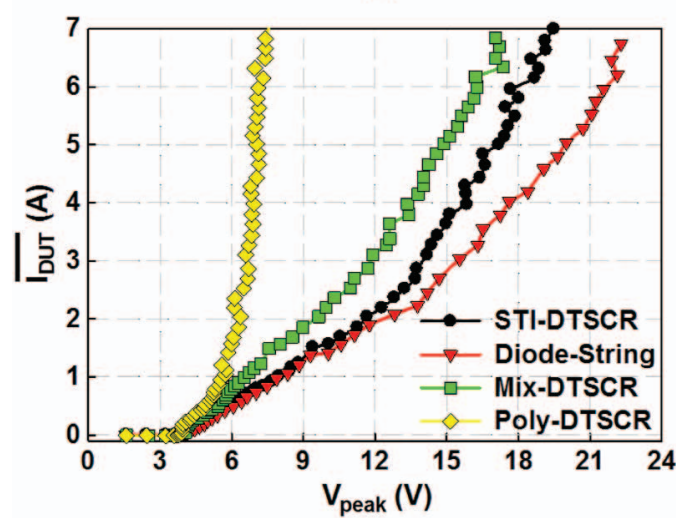

(b)

Fig. 2. (a) Quasi-static $I-V$ characteristics and (b) peak overshoot voltage versus quasi-static current for the different test structures. Data extracted from vf-TLP.

Two additional observations are made in regard to the data in Fig. 2(b). First, the Mix-DTSCR provides only marginally better voltage clamping than does the STI-DTSCR. Defining $V_{\mathrm{OV}}=V_{\text {peak }}-\overline{V_{\mathrm{DUT}}}$, the Mix-DTSCR provides only about a $2-\mathrm{V}$ reduction in $V_{\mathrm{OV}}$ at $7 \mathrm{~A}$. This result indicates that, in the Mix-DTSCR, the STI-bound diode $\mathrm{D}_{1}$ dominates the total impedance of the current path from $\mathrm{D}_{1}$ to $\mathrm{D}_{4}$, at least on the subnanosecond time scale.

Second, the $I-V$ curve for the Mix-DTSCR shows a gradual slope change starting at around $12 \mathrm{~V}$, which is the n-well/ p-well junction breakdown voltage; this is attributed to avalanche-generated electrons flooding the n-well of $\mathrm{D}_{1}$ and reducing its on-resistance. This effect is not seen in the $I-V$ curves of the STI-DTSCR and the Diode-String because their on-resistances are dominated by the STI-bound diodes $\mathrm{D}_{2}, \mathrm{D}_{3}$, and $\mathrm{D}_{4}$; in contrast, the $I-V$ curves for these devices undergo a sudden slope change at about $13.5 \mathrm{~V}$. This has been attributed to n-well/p-well junction breakdown followed by the triggering of the parasitic SCR between the $\mathrm{p}^{+}$of $\mathrm{D}_{1}$ and the $\mathrm{n}^{+}$of $\mathrm{D}_{4}$, which provides an additional current path in parallel with the trigger circuit [14]; the parasitic SCR is triggered at a voltage higher than the $12-\mathrm{V}$ junction breakdown voltage because each diode in the DTSCR is surrounded by a $\mathrm{p}^{+}$guard ring [see Fig. 1(b)] [20]. The Diode-String's quasi-static $I-V$ curve [Fig. 2(a)] similarly undergoes a slope change at $13.5 \mathrm{~V}$.

In Fig. 3, $V_{\mathrm{DUT}}(t)$ for all three DTSCRs is plotted for pulses with $\overline{I_{\mathrm{DUT}}}=2 \mathrm{~A}$. The voltage across the Poly-DTSCR 


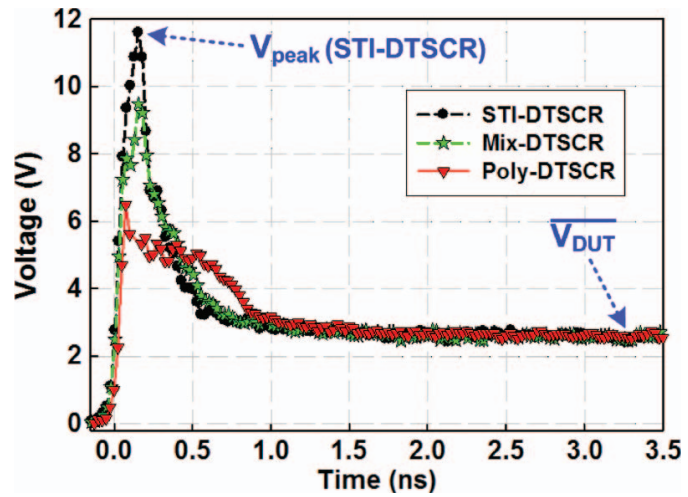

Fig. 3. VF-TLP. Measured voltage waveforms for different DTSCRs.

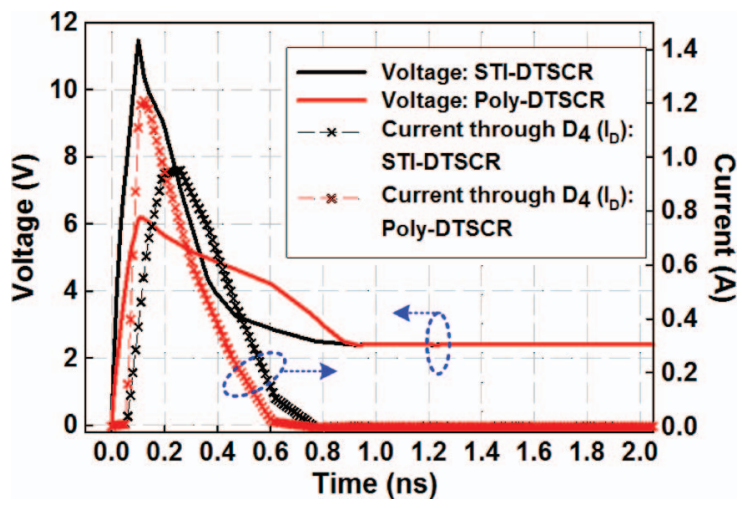

Fig. 4. Simulated transient waveforms for STI-DTSCR and Poly-DTSCR.

is clamped to $6 \mathrm{~V}$, whereas it nearly reaches $10 \mathrm{~V}$ in the Mix-DTSCR and exceeds $11 \mathrm{~V}$ in the STI-DTSCR. However, the Poly-DTSCR reaches its steady-state voltage more slowly, with $V_{\mathrm{DUT}}(t)$ showing a shoulder rather than collapsing rapidly toward its final value. In fact, between 0.5 and $0.9 \mathrm{~ns}$, the voltage across the Poly-DTSCR is higher than that across the STI-DTSCR or the Mix-DTSCR. To understand this result, the transient responses of STI-DTSCR and Poly-DTSCR were simulated using Cadence Virtuoso Spectre. The trigger diodes are modeled using the same approach presented in [21]. The SCRs are modeled as cross-coupled n-p-n and p-n-p transistors; model parameters are provided by the foundry based on the Gummel-Poon BJT model. The simulation results are shown in Fig. 4; the simulated $V_{\text {DUT }}(t)$ looks quite similar to $V_{\text {DUT }}(t)$ obtained from vf-TLP measurement. The simulated current in $\mathrm{D}_{4}$ (i.e., through the diode string), $I_{D}$, is also plotted in Fig. 4. $I_{D}$ for the STI-DTSCR has a slower rise time than that for the Poly-DTSCR, and $I_{D}$ continues to flow at later time points in the STI-DTSCR. $I_{D}$ augments current flow through the SCR, thereby helping to reduce the voltage at the anode; a faster turnoff of $I_{D}$ removes the supplemental current path. In the Poly-DTSCR, once the diode string turns off, the decay of the anode voltage is determined solely by the intrinsic SCR and is a relatively slow process. Despite the shoulder in its $V_{\mathrm{DUT}}(t)$, the Poly-DTSCR is still the best CDM ESD protection device among the investigated DTSCRs due to its substantially reduced peak voltage.

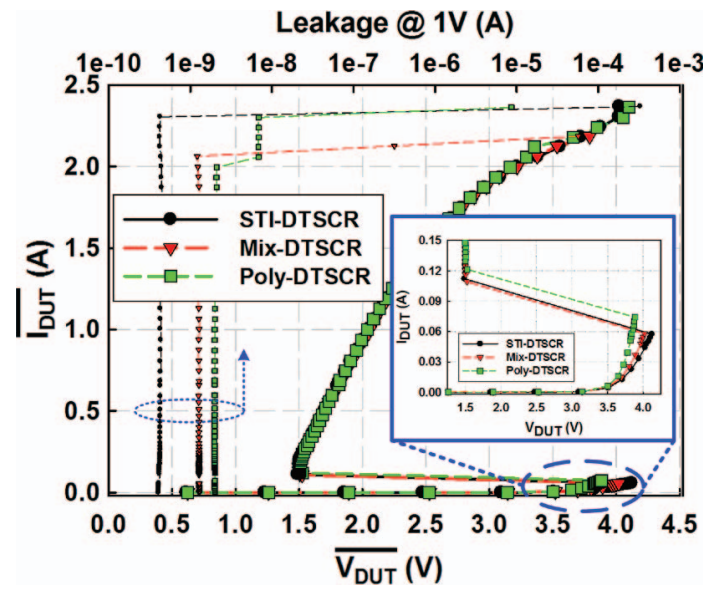

Fig. 5. Quasi-static $I-V$ characteristics of different DTSCRs, extracted from 100-ns TLP.

TABLE II

MeAsurement Results of DifFEREnt DTSCRS

\begin{tabular}{|c|c|c|c|c|c|c|}
\hline & Vf-TLP & \multicolumn{3}{|c|}{$100-\mathrm{ns} \mathrm{TLP}$} & \multirow{2}{*}{$\Delta C$} & $\begin{array}{c}\text { Leakage } \\
\text { @ 1V }\end{array}$ \\
\cline { 2 - 5 } & $\mathrm{V}_{\mathrm{OV}} @ 7 \mathrm{~A}$ & $\mathrm{I}_{\mathrm{t} 1}$ & $\mathrm{~V}_{\mathrm{t} 1}$ & $\mathrm{I}_{\text {fail }}$ & & (120 pA \\
\hline STI-DTSCR & $12.6 \mathrm{~V}$ & $57.3 \mathrm{~mA}$ & $4.12 \mathrm{~V}$ & $2.36 \mathrm{~A}$ & - & 420 \\
\hline Mix-DTSCR & $10.6 \mathrm{~V}$ & $58 \mathrm{~mA}$ & $4.02 \mathrm{~V}$ & $2.12 \mathrm{~A}$ & $+16 \mathrm{fF}$ & $1.27 \mathrm{nA}$ \\
\hline Poly-DTSCR & $1.5 \mathrm{~V}$ & $74.1 \mathrm{~mA}$ & $3.88 \mathrm{~V}$ & $2.06 \mathrm{~A}$ & $+37 \mathrm{fF}$ & $2 \mathrm{nA}$ \\
\hline
\end{tabular}

\section{Additional Performance Metrics}

Quasi-static $I-V$ characteristics obtained from 100-ns TLP [22] measurements are shown in Fig. 5. Because all DTSCRs have the same anode-to-cathode spacing, all three DTSCRs have the same holding voltage of $1.5 \mathrm{~V}$. The trigger voltage and current $V_{t 1}$ and $I_{t 1}$ are obtained from the data shown in the figure inset. The failure current $\left(I_{\text {fail }}\right)$ is defined as the TLP current which causes the leakage (measured at $1 \mathrm{~V}$ ) to increase by more than $10 \%$. The test structure layout did not permit for measurement of the device S-parameters, which would allow for accurate measurement of the capacitance with the bondpads and other parasitics de-embedded. However, using an $L C R$ meter to measure the total capacitance of each structure, one can easily observe the incremental increase in capacitance as the trigger diodes are changed from STI-bound to polybound. The values of $V_{\mathrm{OV}}, V_{t 1}, I_{t 1}, I_{\mathrm{fail}}$, and $C$ are listed in Table II. The Poly-DTSCR has slightly reduced $I_{\text {fail }}$, slightly increased capacitance, and greatly increased leakage $(5 \times)$ relative to the STI-DTSCR.

The dc $I-V$ characteristics of stand-alone diodes are shown in Fig. 6. Below $0.6 \mathrm{~V}$, the gate leakage current dominates the junction leakage current, and the poly-bound diode conducts more current than the STI-bound diode. The large leakage current of poly-bound diodes explains why the Poly-DTSCR has higher leakage current than the STI-DTSCR. The polybound diodes used in this work each have their gate tied to their anode, i.e., the $\mathrm{p}^{+}$side. The measurement results in Fig. 6 suggest that the leakage current of a Poly-DTSCR can likely be reduced below the value shown in Table II simply by changing the gate connection from the anode to the cathode 


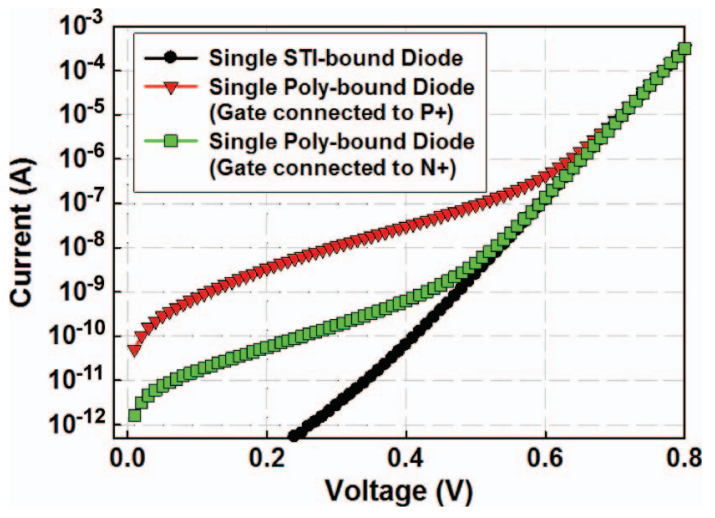

Fig. 6. DC $I-V$ characteristics of a single STI-bound diode and a single polybound diode with varying gate connections. Both diodes have the same p-n junction perimeter of $200 \mu \mathrm{m}$.

$\left(\mathrm{p}^{+}\right.$to $\left.\mathrm{n}^{+}\right)$. Measurement results indicate that such a change also reduces the capacitance of the diode and does not affect its ESD performance, results confirmed by the data in [23] and [24].

\section{CONCLUSION}

The trigger circuit used with an SCR-based ESD protection device determines the magnitude of the voltage overshoot that occurs before the SCR fully turns on. Poly-bound diodes exhibit reduced voltage overshoot relative to STI-bound diodes; this work demonstrates that, when poly-bound diodes are integrated into a DTSCR, the resulting structure provides very good voltage clamping (an experimental finding recently confirmed by others [25]). All the diodes in the trigger circuit, including the diode intrinsic to the SCR, must be poly-bound to obtain the benefit.

Depending on whether low power or high speed is the design objective, either an STI-DTSCR or a Poly-DTSCR may be the better selection. The STI-DTSCR has significantly lower leakage current and can be augmented by a secondary protection circuit in order to provide CDM ESD protection. However, the secondary protection will reduce the performance of radio-frequency or high-speed input/output circuits. The PolyDTSCR reduces the need for a secondary protection at the cost of increased leakage current.

\section{ACKNOWLEDGMENT}

Test structures were fabricated under the TSMC University Shuttle Program.

\section{REFERENCES}

[1] M.-D. Ker and K.-C. Hsu, "Overview of on-chip electrostatic discharge protection design with SCR-based devices in CMOS integrated circuits," IEEE Trans. Device Mater. Rel., vol. 5, no. 2, pp. 235-249, Jun. 2005.

[2] M.-D. Ker and K.-C. Hsu, "SCR device fabricated with dummy-gate structure to improve turn-on speed for effective ESD protection in CMOS technology," IEEE Trans. Semicond. Manuf., vol. 18, no. 2, pp. 320-327, May 2005 .

[3] M. Mergens, C. Russ, K. Verhaege, J. Armer, P. Jozwiak, R. Mohn, B. Keppens, and C. Trinh, "Speed optimized diode-triggered SCR
(DTSCR) for RF ESD protection of ultra-sensitive IC nodes in advanced technologies," IEEE Trans. Device Mater. Rel., vol. 5, no. 3, pp. 532-542, Sep. 2005.

[4] J. Di Sarro, K. Chatty, R. Gauthier, and E. Rosenbaum, "Study of design factors affecting turn-on time of silicon controlled rectifiers (SCRs) in 90 and $65 \mathrm{~nm}$ bulk CMOS technologies," in Proc. IEEE Int. Rel. Phys. Symp., 2006, pp. 163-168.

[5] T. Smedes and N. Guitard, "Harmful voltage overshoots due to turn-on behaviour of ESD protections during fast transients," in Proc. EOS/ESD Symp., 2007, pp. 357-365.

[6] C. Chu, A. Gallerano, J. Watt, T. Hoang, T. Tran, D. Chan, W. Wong, J. Barth, and M. Johnson, "Using VFTLP data to design for CDM robustness," in Proc. EOS/ESD Symp., 2009, pp. 286-291.

[7] N. Jack and E. Rosenbaum, "ESD protection for high-speed receiver circuits," in Proc. IEEE Int. Rel. Phys. Symp., 2010, pp. 835-840.

[8] J. Wu, P. Juliano, and E. Rosenbaum, "Breakdown and latent damage of ultra-thin gate oxides under ESD stress conditions," in Proc. EOS/ESD Symp., 2000, pp. 287-295.

[9] A. Ille, W. Stadler, A. Kerber, T. Pompl, T. Brodbeck, K. Esmark, and A. Bravaix, "Ultra-thin gate oxide reliability in the ESD time domain," in Proc. EOS/ESD Symp., 2006, pp. 285-294.

[10] D. Ellis, Y. Zhou, J. Salcedo, J.-J. Hajjar, and J. Liou, "Prediction and modeling of thin gate oxide breakdown subject to arbitrary transient stresses," IEEE Trans. Electron Devices, vol. 57, no. 9, pp. 2296-2305, Sep. 2010.

[11] G. Wybo, S. Verleye, B. Van Camp, and O. Marichal, "Characterizing the transient device behavior of SCRs by means of VFTLP waveform analysis," in Proc. EOS/ESD Symp., 2007, pp. 366-375.

[12] J. Bourgeat, C. Entringer, P. Galy, P. Fonteneau, and M. Bafleur, "Local ESD protection structure based on silicon controlled rectifier achieving very low overshoot voltage," in Proc. EOS/ESD Symp., 2009, pp. 314-321.

[13] K. Watanabe, T. Hiraoka, T. Sei, and K. Numata, "New protection techniques and test chip design for achieving high CDM robustness," in Proc. EOS/ESD Symp., 2008, pp. 332-338.

[14] R. Gauthier, M. Abou-Khalil, K. Chatty, S. Mitra, and J. Li, "Investigation of voltage overshoots in diode triggered silicon controlled rectifiers (DTSCRs) under very fast transmission line pulsing (VFTLP)," in Proc. EOS/ESD Symp., 2009, pp. 334-343.

[15] I. Ladany, "An analysis of inertial inductance in a junction diode," IRE Trans. Electron Devices, vol. 7, no. 4, pp. 303-310, Oct. 1960.

[16] M.-D. Ker, K.-H. Lin, and C.-H. Chuang, "MOS-bounded diodes for on-chip ESD protection in a $0.15-\mu \mathrm{m}$ shallow-trench-isolation salicided CMOS process," in Proc. IEEE Int. Symp. VLSI Technol. Syst. Appl., 2003, pp. 84-87.

[17] J.-R. Manouvrier, P. Fonteneau, C.-A. Legrand, P. Nouet, and F. Azais, "Characterization of the transient behavior of gated/STI diodes and their associated BJT in the CDM time domain," in Proc. EOS/ESD Symp., 2007, pp. 165-174.

[18] E. Grund and R. Gauthier, "VF-TLP systems using TDT and TDRT for Kelvin wafer measurements and package level testing," in Proc. EOS/ESD Symp., 2004, pp. 338-345.

[19] S. Malobabic, J. Salcedo, J.-J. Hajjar, and J. Liou, "Analysis of safe operating area of NLDMOS and PLDMOS transistors subject to transient stresses," IEEE Trans. Electron Devices, vol. 57, no. 10, pp. 2655-2663, Oct. 2010.

[20] U. Glaser, K. Esmark, M. Streibl, C. Russ, K. Domanski, M. Ciappa, and W. Fichtner, "SCR operation mode of diode strings for ESD protection," in Proc. EOS/ESD Symp., 2005, pp. 60-69.

[21] M. Stockinger, S. Ruth, J. Miller, K. Nguyen, Y. Akrout, M. Kearney, B. Drew, and S. Ngo, "CDM protection design for CMOS applications using RC-triggered rail clamps," in Proc. EOS/ESD Symp., 2009, pp. 91-100.

[22] A. Gerdemann, K. Bhatia, and E. Rosenbaum, "A Kelvin transmission line pulsing system with optimized oscilloscope ranging," in Proc. EOS/ESD Symp., 2007, pp. 80-88.

[23] S. Cao, A. Salman, J.-H. Chun, S. Beebe, M. Pelella, and R. Dutton, "Design and characterization of ESD protection devices for high-speed I/O in advanced SOI technology," IEEE Trans. Electron Devices, vol. 57, no. 3, pp. 644-653, Mar. 2010.

[24] S. Voldman, S. Geissler, J. Nakos, J. Pekarik, and R. Gauthier, "Semiconductor process and structural optimization of shallow trench isolationdefined and polysilicon-bound source/drain diodes for ESD networks," in Proc. EOS/ESD Symp., 1998, pp. 151-160.

[25] J. Li, R. Gauthier, J. Di Sarro, and R. Mishra, presented at the Int. Electrostatic Discharge Workshop, 2011. 


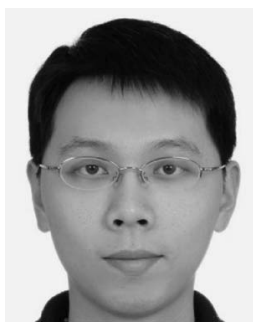

Wen-Yi Chen (S'03) received the B.S. degree from the Department of Electronics Engineering, National Chiao Tung University, Hsinchu, Taiwan, in 2003 and the M.S. degree from the Institute of Electronics, National Chiao Tung University, in 2005, where he is currently working toward the Ph.D. degree.

After military service, he joined the Circuit Design Department, SoC Technology Center, Industrial Technology Research Institute, Hsinchu, as a Circuit Design Engineer. In 2006, he joined the Amazing Microelectronic Corporation, where he worked with system-level electrostatic discharge (ESD) protection design. His current research interests include reliability of high-voltage CMOS devices and ESD protection design in mixed-voltage $\mathrm{I} / \mathrm{O}$ circuits.

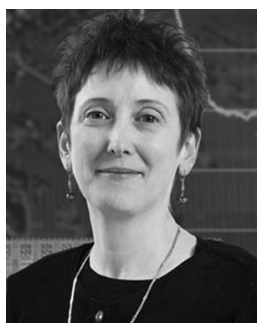

Elyse Rosenbaum (F'11) received the B.S. degree (with distinction) in electrical engineering from Cornell University, Ithaca, NY, in 1984, the M.S. degree in electrical engineering from Stanford University, Stanford, CA, in 1985, and the Ph.D. degree in electrical engineering from the University of California, Berkeley, in 1992.

From 1984 to 1987, she was a Member of Technical Staff with AT\&T Bell Laboratories, Holmdel, NJ. She is currently a Professor with the Department of Electrical and Computer Engineering, University of Illinois, Urbana. She has been a Visiting Professor with Katholieke Universiteit, Leuven, Belgium, and National Chiao Tung University, Hsinchu, Taiwan. She has authored or coauthored over 100 technical papers. She has given invited lectures at many universities and industrial laboratories. Her present research interests include design, testing, modeling, and simulation of electrostatic discharge (ESD) protection circuits, ESD reliability of stacked packaging, design of high-speed circuits with ESD protection, latch-up, gate oxide degradation, and substrate noise coupling.

Dr. Rosenbaum was a recipient of a Best Student Paper Award from the IEDM, a Technical Excellence Award from the SRC, a NSF CAREER Award, a IBM Faculty Award, and a UIUC Bliss Faculty Scholar Award. She is an Editor for IEEE Transactions on Device and Materials Reliability. She has presented tutorials on reliability physics at the International Reliability Physics Symposium, the EOS/ESD Symposium, and the RFIC Symposium. She was the Keynote Lecturer at the 2004 Taiwan ESD Conference.

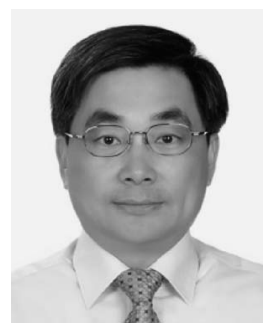

Ming-Dou Ker (S'92-M'94-SM'97-F'08) received the $\mathrm{Ph} . \mathrm{D}$. degree from the Institute of Electronics, National Chiao Tung University, Hsinchu, Taiwan, in 1993.

He was the Department Manager with the VLSI Design Division, Computer and Communication Research Laboratories, Industrial Technology Research Institute, Hsinchu. Since 2004, he has been a Full Professor with the Department of Electronics Engineering, National Chiao Tung University. During 2008-2011, he was rotated to be the Chair Professor and the Vice President of I-Shou University, Kaohsiung, Taiwan. He is currently a Distinguished Professor with the Department of Electronics Engineering, National Chiao Tung University. During 2010-2011, he was the Executive Director of the National Science and Technology Program on System-onChip, Taiwan. He is currently the Executive Director of the National Science and Technology Program on Nano Technology, Taiwan (2011-2014). In the technical field of reliability and quality design for microelectronic circuits and systems, he has published over 400 technical papers in international journals and conferences. He has proposed many solutions to improve the reliability and quality of integrated circuits, which have been granted with 187 U.S. patents and 162 Taiwan patents. He had been invited to teach and/or to consult the reliability and quality design for integrated circuits by hundreds of design houses and semiconductor companies in the worldwide IC industry. His current research interests include reliability and quality design for nanoelectronics and gigascale systems, high-speed and mixed-voltage I/O interface circuits, onglass circuits for system-on-panel applications, and biomimetic circuits and systems for intelligent prosthesis.

Dr. Ker has served as a member of the Technical Program Committee and the Session Chair of numerous international conferences for many years. He served as the Associate Editor for the IEEE TRANSACTIONS ON VLSI SYSTEMS in 2006-2007. He was selected as the Distinguished Lecturer in the IEEE Circuits and Systems Society (2006-2007) and in the IEEE Electron Devices Society (2008-present). He was the President of Foundation in Taiwan ESD Association. In 2009, he was awarded as one of the top ten distinguished inventors in Taiwan. 